\title{
Building Rubik's Cube: A Function Of Production
}

\author{
José Villacís González, (Email: villacis.fhm@ceu.es), Universidad San Pablo, Spain
}

\begin{abstract}
The Rubik's cube is a special game and a very particular puzzle. The 3-dimensional cube is made up of six faces, or boundary sections, of the same size. Each face, or section, consists of several two dimensional square parts, or cubelets. Every cubelet has the same surface area, and each of the six faces has the same number of cubelets. Therefore, the cube's surface is entirely covered with isocubelets. The cubelets are painted in six different colours, and it is possible to create a design where each face shows only one colour. Such is the object of the game: to turn the cubelets and sections of the cube so that only one (different) colour shows on each one of the six faces. If one manages to master the puzzle, the cube will show six faces of the same size, each coloured differently. The cubelets and sections of the cube can be turned both horizontally and vertically in order to change colours while trying to determine the appropriate combination to complete the puzzle. This approach is linked to a particular function in microeconomics that deals with the relationship between two magnitudes: on the one hand, the moves needed to achieve the desired final design; and on the other hand, the cost linked to the required production processes. This analytical model must use combinatorial mathematics' equipment because, after all, the key factor in solving the Rubik's cube is the way in which the cubelets and sections are arranged.
\end{abstract}

\section{Introduction}

reating a certain design on the Rubik's cube from a situation of total disorder, or a situation of perfect disorder, entails a series of horizontal and vertical rotating moves; the only possible moves, aimed at positioning cubelets of the same colour to the same face. Therefore, it is essential to know the number of moves required to fully arrange the cube. To appreciate this operation, it is important to first understand some basic premises.

The first premise states that in Rubik's cube there is only one possible logical and mechanical move per turn. According to this, each move shapes a different map in the cube, and this map indicates whether one is approaching the final solution, and at the same time it indicates new problems to be faced for subsequent moves.

The second premise is a matter of deciding a goal and asking oneself what type of arrangement one prefers. In this sense, we should decide two goals: one total solution and a few partial solutions (or just one partial solution). One total solution requires that the cube shows an even colour on all six faces, and that each face shows a different colour, which means that every face must be put together with cubelets of the same colour. Partial solutions, or goals, seek to manipulate the puzzle such that one, or several (but not all), faces show the same colour. Alternately, partial solutions include those that seek to have one, or several faces, aligned with one or more lines of cubelets of the same colour.

If we add an arbitrary effort value to each move, measured in quantum units for instance, we will be in a position to measure the total, partial, average and marginal cost of building Rubik's cube with a specific purpose. At this point, it is important to understand that putting part of a line together or a full line or a face - which is a group of lines - or putting several faces together, represents a specific combination of consecutive cubelets. Putting several consecutive cubelets of the same colour together will fully or partially determine a line; it will determine a full line or a segment of a line. 
In the end, we see that a combinatorial mathematical treatment is necessary from the beginning; a combinatorial mathematical treatment, which in fact corresponds from beginning to end, to the problem of building Rubik's cube.

We must clarify that when we mention the building of Rubik's cube we do not mean physically creating it, since the cube is already assembled and all one does is play with the colours to create different designs (full lines or segments of lines).

\section{Combinatorial Math}

Placing several elements in an ordered sequence requires a special situation within a range of possible combinations. This corresponds to the task of counting and enumerating, the combination of every possible ordering. This task is the concern of combinatorial mathematics. The general expression of combinatorial math is used to refer to all instances of arrangement and combination, including the very same special case of combinatorial math.

Any issue related to consumption and production microeconomics should necessarily and initially deal with the arrangement of the elements (goods or factors) and should, therefore, apply combinatorial theory. In consumption, every different way of arranging goods provides a different level of utility - provided that we do not consider the case of one or more combinations generating the same utility. For the purposes of our paradigmproduction (not the cube but the game of the cube)- each arrangement, or sequence, of elements decides the production volume - provided that we do not consider the case of one or more combinations generating the same volume.

Having justified the combinatorial treatment of our paradigm, we will proceed to mathematical analysis. When we mention production in this particular game theory, we mean total or partial results obtained in lines or faces.

In principle, every element of the cube, or $\boldsymbol{n}$, will be included in the game. There cannot be more than $n$ elements (it is physically and theoretically impossible) and there cannot be less than $n$ elements (it is physically impossible and theoretically rejectable). If we take all of the elements $(n)$ into consideration for the game, it is with the aim of studying our paradigm in full; its purpose being to paint six faces, each with different colours. Once we have analyzed this general and difficult case, we will proceed to analyze special cases where the number of elements is $n^{\prime}$, where $n^{\prime}<n$. This would correspond to the treatment needed to build lines of the same colour, or even to paint one or more faces (not all).

We will consider a set $\mathbf{A}$ of $n$ elements (cubelets) of order $n$, each order containing every element in $A$ (which integrates the cube). Combinatorial theory calls this set an ordinary permutation, represented by $P_{n}$. Consequently, in ordinary permutations, all the elements are included and considered. Also, the elements are arranged in every possible way, although none is repeated. Moreover, the number of cubelets (elements) is limited, fixed, and are not repeated, and each cubelet is irreplaceable. For instance, one yellow cubelet is a different element than another yellow cubelet.

The number of possible orderings, or ordinary permutations, is determined by factorial of $n$ elements:

$n !$

Rubik's cube has nine cubelets on each face arranged in three lines. Since there are six faces, the total number of cubelets is $54(9 * 6=54)$. If we apply the above formula, then the number of possible combinations would be $(n=54)$ :

$54 !=54.53 .52$ 
The fact that these are all possible combinations does not mean that such number determines the combination sought. The combination sought is one, and only one, in the whole set.

\section{Probability}

Ordinary permutations bring us to the theory of probability, and the theory of probability takes us to the theory of production. If we throw several cubes in a giant box, and each cube shows a specific sequence or ordering, all of them different from one another, then there would be 54! cubes in total. It is obvious that the combination we are looking for will be among all those cubes. We will now have to randomly choose a cube, any cube, and calculate the probability that it corresponds to our sought after combination.

First, we know that such combination, which is one and only one, will be smaller than all possible combinations. That is: $1<54$ !

Calculating the probability is the quotient of that unit by all possible combinations:

Probability $=1 / n$ !

In our example, it would be:

$1 / 54$ !

A suggestive way of dislocating probability is to continue drawing cubes until the combination we are looking for is pulled out. This statement is viable for the theory of probability, but the macroeconomic safe way consists in calculating, in advance, all of the possible combinations in order to find out how long it takes, or how much it costs, to achieve the sought after combination.

These reflections about probability have inevitably led us to the arduous, but infallible way of creating a specific design on the cube. Such is the question.

\section{Theory Of Disorder}

Building is destroying and, from destruction (disorder), reaching an overall order. This statement is valid for any definition of production. Disorder may have two meanings: one is that of a priori disorder, and the other is that of a posteriori disorder.

In the first case, we deal with the Greek philosophical concept of chaos, which involves a plasmatic and indifferent state of matter with no forces or physical laws, where matter has an indifferent condition. In microeconomics, this would entail a situation where production factors of any kind are motionless, with nobody calling them to produce. In our example, or paradigm, the elements being ignored and no player wanting to participate represent this situation.

The second case would be a situation where production factors are called to produce, consumed and destroyed to engender another reality. As regards to our paradigm, the cube would be in an initial situation where all the elements are in a perfect and complete disorder. Such situation means that 54 ! moves will be necessary in order to combine all the elements (ordinary permutations) to achieve the sought after combination. And if a certain set of moves, enough to comprise 54! combinations, is necessary, it means that the initial disorder is perfect and complete. This is the situation we are analyzing: a posteriori disorder that is complete, for it will help us measure final disorder.

It is very important to bear in mind that if there are 54 ! possible combinations, the number of moves leading to those 54 ! combinations will be higher. If we name each move as $\mathbf{G}$, then $G>n$ ! This means that there will have to be more moves than combinations. In other words, each marginal combination (or next combination) will often follow more than one move. This is one of the Rubik's cube's characteristics. 


\section{Compact Geometry}

In any puzzle, or game, every piece is placed in a specific position within the whole set and is in perfect harmony with the rest of pieces. In the case of a traditional puzzle representing a map, a landscape, a face, or any other design, the pieces have a specific position, which, along with the rest of pieces, serves the purpose of putting the design together.

In the case of Rubik's cube, the position is, geometrically speaking, more oppressive and binding in the sense that every piece determines the position of the other pieces (as in every puzzle) but it also determines, and rigidly structures the moves. This is the reason why we call the cube a compact geometry game. The cube will always be a cube, but each position and move of the cubelets and/or the faces, informs the other cubelets and faces, and we may even say that they get them out of order.

Contrary to a traditional puzzle, with each move of Rubik's cube, a certain situation is put in order, and simultaneously the other faces of the cube are placed out of order. Therefore, new, additional, or marginal efforts will be necessary to bring the cube to the final ordering we seek. In the macroeconomic theory of production, scale diseconomies occur as the game progresses towards the final ordering, which is also contrary to what happens in a traditional puzzle. These scale diseconomies generated in production require more than proportional effort units to offset them, which means that marginal costs and production means will increase. In traditional puzzles, the effort decreases as the puzzle is shaped and progression is faster. The case of the Rubik's cube is exactly the opposite.

If we know all possible combinations in a cube (n!), it is very difficult to know all the moves needed to achieve the final situation where a specific combination corresponds to the final ordering we seek. Yet, because in this peculiar function of production (scale diseconomies), difficulties arise as the final ordering is achieved, it becomes obvious that the number of moves also increases, and they always increase in a number higher than all combinations taken together. As stated above: $G>n$ !

We cannot venture multiplying $\mathrm{k}$, such that $n ! k=G$, where $k>1$, because we are not sure that it is a constant, but a multiplication where the multiplier increases in value. This line of reasoning is developed and dealt with in greater detail in Section 6, below.

\section{A Geometric Progression Of Moves And Difficulties}

Before we continue with this mathematical topic, we need to clarify two points. First, we should ask ourselves about the nature of moves: What are we referring to, to all of the moves, or to every single and last move? Secondly, we should know the difficulty growth rate -considering that the player does not have a handbook available with the final solution. The answer to the first question is that we refer to the last move, or marginal move. This answer leads to the following assertion: we will be able to determine all of the moves.

To answer the second question, we will say that moves follow an increasing pace similar to an increasing geometric progression -always taking into account, we insist, that the player ignores the final solution, or the only efficient way. As one progresses, or one puts the cubelets in place, a greater risk arises of placing the other cubelets which were in order - out of order, and therefore extra effort and moves are needed to avoid this increased disorder. Now, is it necessary to take into account the fact that difficulties escalate? We could answer by saying that the first cubelet arrangements moved on the cube are easy, since they do not necessarily affect other cubelet arrangements, simply because no such other arrangements exists. Consequently, the answer is yes: a positive geometric progression of difficulties is to be considered and so, the costs related to that progression will increase at the same pace. Such progression will eventually stop; it is not unlimited.

These assertions are valid provided that the player has not received any previous instructions and that he/she starts from a situation of perfect disorder. 


\section{Reason, Strategy And Chance In Building}

The line of argument we have developed thus far, carries an uncertain or hazy aspect, which, in general, is present in any puzzle-like game: the combination of rationality, strategy and chance. Guided by reason, we can know a perfect disorder situation and the final ordering situation that we are looking for, and in these circumstances, reason searches for the necessary solution. Strategy, which is a manoeuvre of reason, plays an important role. Since we do not have a handbook directly leading us to the final solution, strategy carries out intuitive searches based on mistakes, in order to find a direction avoiding fruitless paths. This strategic exclusion of paths does not mean that we know the only efficient way to the final solution (if the player knew everything, there would be no game). It is simply a question of disregarding absurd ways. Chance is always present in the game, for good or for bad, but not too prominently, because after all, it is the logical construction of a geometric structure. The role of chance in poker and in chess is different, although both use reason and strategy. Chance has a greater influence on the hand of cards dealt in poker than on chess.Strategy, and chance, to a lesser extent, is involved in the function of building Rubik's cube, determining its shape and representation on the curve of average and marginal costs.

If strategy and chance have an influence, learning has an even greater capacity for involvement, and will carry weight in our work. There are many types of puzzles in the sense that there are many maps or drawings to make; and, to a certain extent, though not completely; at first the player is a beginner. However, there is only one Rubik's cube, at least for now, although according to mathematical and geometric theory, it is possible to build complex cubes like Rubik's. We will not consider such possibility. A veteran Rubik's cube player will know the short ways to achieve the final ordering goals. In this sense, he/she will elude, thanks to his/her experience, the power of scale diseconomies, and even avoid the appearance of increasing average and marginal costs.

\section{Combinations And Final Ordering}

As we have seen, the final ordering we want to achieve in the cube can be a full ordering, where all six faces show a specific colour, that is, the cube is painted in six different colours, one per face. We can also try a partial result: one or more faces (never six) showing a specific colour, or one or more lines showing the same colour.

In theory, by applying combinatorial theory, and more specifically, ordinary permutations, every possible ordering should be explored - measured by $n !$ - and we should choose our sought after combination from among all possibilities. Though possible in theory, in practice is avoided, basically due to an economic concern: the management of limited resources, which, in this case, are the moves leading to the final solution. It would be a waste to search for all the combinatorial moves in order to reach and choose the final move we are looking for. It is as if a guest ate all the dishes on the menu in every possible order so as to declare at the end that this or that combination is the one providing the higher level of satisfaction. There will be efficient players and less efficient players, and even bad players, but all of them will gradually try different orderings, different ways; they will make mistakes and little by little, with more or less difficulty, will come closer to the final ordering desired until they achieve it. Yet, in doing so, they will not use up every possible combination (permutations).

At this point, we wonder from a theoretical approach, if all $n$ ! combinations are necessary to achieve the goal we are looking for: the full goal or partial goals (just one or more faces or lines only (one or more lines)). We may say that the answering matrix, which is not an economic matrix but a mathematical one, is all possible combinations (ordinary permutations) counted as $n$ !. However, each case, that is, each goal, involves considering special ordinary permutations made up by groups of different elements included in $n$.

We will name the colours as $n^{\prime}$ groups, such that $n^{\prime}$ belongs to $n$ and, therefore: $n^{\prime}<$ n. Since there are six colours, then: $6 n^{\prime}=n$.

Consequently, if we want to play with just one colour and paint just one face, the possible orderings will be $n^{\prime}$ ! If we play with two colours and two faces: $2 n^{\prime}$ !, and so on. If we want to go for the full game, then: $6 n^{\prime} !$ is $n$ ! $\left(6 n^{\prime} !=n !\right)$. 
If we focus on just one line, it will be worth three, which is a smaller value than 9 , since 9 correspond to all of the cubelets of the same colour. In other words: $n^{\prime \prime}<n^{\prime}<n$. We will name three cubelets as $n^{\prime \prime}$ (three is the number of cubelets that define a line). Therefore, the number of possible combinations needed to achieve at least one line would be $n^{\prime \prime}$ ! How many lines are there on a face? Three lines: since each line is made up of three cubelets, then three lines will be needed to complete a face, which is made up of nine cubelets $(3 * 3=9)$.

We could also raise the following question: how many combinations are needed to achieve at least one face? The answer would be: $3 n^{\prime \prime}$ ! Furthermore, we could ask: how many combinations are needed solve the puzzle, or to achieve the full cube, with each face painted in a different colour? Here, we would first have to ask how many lines are there in a cube. The answer is 18, since there are three lines per face and the cube has six faces. However, it is important to indicate that it is possible to have three lines of the same colour per face.

In short:

$n^{\prime \prime}<n^{\prime}<n$

$6 n^{\prime}=n$

$3 n^{\prime \prime}=n^{\prime}$

Therefore: $18 n^{\prime \prime}=n$

\section{Most Efficient Way Or Ideal Route}

Let's go back to our initial reflections about not needing to explore all combinations to achieve the desired goal but, on the contrary, that the aim of an economy would be to elude most of those combinations. In other words, seeking economic efficiency means, precisely, avoiding useless combinations. Following useless ways that do not lead to the final result represents waste in the production function. This idea can be expressed as follows: looking for the most, or least efficient combinations requires achieving approximately the maximum possible production at the minimum possible cost. We believe such is the definition of the significant facts in economic terms.

To deal with the issue of efficiency, which is the issue of following an ideal route, we must go back to the question of rationality, strategy and chance, and most of all, learning.

Efficiency is, by definition, a term used by economists virtually to define economy. It essentially means achieving the maximum production with the minimum effort, that is, with the minimum cost. In the process of achieving a final goal, whether it is total or partial, we call efficient ways those that make it possible to achieve the final result with the least moves, and so the minimum effort. This statement is directly related to total combinations, since total combinations, show to their full extent, exactly the opposite of efficiency: the result is achieved with the maximum effort or possible efforts. Therefore, moves based on possible combinations indicate a maximum or pure inefficiency. If we compare the sense of maximum efficiency with that of maximum inefficiency, we obtain a maximum savings of moves, or possible costs, in the process of achieving the final goals when solving Rubik's cube.

There may be different ways that offer the same maximum efficiency. We mean to say that there are several ways, involving the same number of moves (the minimum number of moves), in order to achieve the final goal, and therefore there will be several combinations, which, like subgroups, represent the idea of efficiency within the total combinations.

Reaching the most efficient way requires a handbook with the answers, or a knowledgeable player who knows the direct way to reach the final goal, or both. Both the handbook and the experienced player should know and start from the initial perfect disorder. Players who are not knowledgeable and do not have a handbook may totally or partially compensate their ignorance with rationality, by perfecting their strategy and/or if they are fortunate and trust chance. They may also compensate such ignorance with experience, which means increasing their knowledge. 
If we consider the knowledgeable player someone capable of being efficient and achieving the most efficient way, he/she will build Rubik's cube like an efficient production function - in the formal sense or in the sense of a game. At this point, we wonder whether scale diseconomies appear as one progresses in building the cube, as we have seen in previous sections. We have seen that, as one works with different orderings, the arrangement of new elements generates disorder in other areas of the cube, and thus causes marginal inefficiencies.

Do these diseconomies occur when a knowledgeable player or someone with a handbook plays? The answer is no, since by definition, the most efficient way itself is a way involving the minimum number of moves required to reach the final destination. Thus the appearance of scale diseconomies indicates the level of ignorance or lack of skill of the player. And if scale diseconomies translate into cost increases, then increasing marginal costs indicate marginal levels of ignorance. The opposite is also true: an improvement of information - learning through experience, for instance - allows for a reduction in costs. Because by definition a variation in costs per production unit (per ordering or arrangement in our case) corresponds to marginal costs, then a variation in experience per game unit causes, in a positive sense, a reduction in marginal costs, and, in a negative sense, an increase in marginal costs. It is at this stage of the learning process that one reaches the top of the ladder, or total knowledge, a situation where scale diseconomies disappear, as well as the increasing tendency for marginal costs.

\section{Conclusion}

We perform a productive activity consisting in the formal building of Rubik's cube by playing "wit the cubelets and colours." This productive activity is a particular production function leading to the knowledge of total, average and marginal costs linked to production volume. Developments in the homogeneous building of the faces, whether totally or partially, or of the lines, are called marginal production volumes, and the complete building of the cube is called total production volume.

Similar to any puzzle game, the object of Rubik's cube is to put the pieces in sequence, in a specific order that cannot be repeated. This requires the use of combinatorial mathematics equipment. Since we consider all possible orderings, where all the elements are present and no element is repeated, when we mention the word combination, we are referring to ordinary permutations. Contrary to a traditional puzzle of a map or a drawing, where the moves get faster as one progresses because the map or drawing appears gradually, in Rubik's cube the situation becomes more complicated as the player progresses. This is due to the destroying tangential effect of other arrangements that occur when new moves are made. Therefore, it is necessary to make additional moves to offset these scale diseconomies, thus causing an increase in total and marginal costs.

There is, in theory, an ideal route, or efficient way, the best among the best, through which it is possible to achieve the final goal; starting from perfect disorder, with no less and no more moves than needed. Consequently, the sense of economic efficiency will by all means avoid combinations of useless moves to reach the final solution. In this regard, the knowledge a player has acquired through experience can be assessed based on the gradual elimination of useless combinations, which allows him/her to go up the marginal efficiency ladder, which is equivalent to saying that marginal costs are gradually reduced.

\section{References}

1. Arrow, J. K., (1950)A Difficult in the Concept of Social Welfare, Journal of Political Economy, 58,

2. Aumann Robert, J.(1987): Game Theory, in New Palgrave.

3. (1995) Backward Induction and Common Knowledge of Rationality, Games EB repeated Games of Incomplete Information, with M. Maschler.

4. $\quad$ Baumol, W.W. J., (1946) Community Indifference, Review of Economic Studies, 14.

5. Bergson, A., A., (1938): Reformulation of Certain Aspects of Wealfare Economics, Quaterly Journal of Economics, 52.

6. Binmore, Ken, (1999): Game Theory and Social Contract: Playing Fair, MIT Press.

7. Debreu, G., (1951) The Coeficient of Resource Allocation, in Ecta, 19, pp 273-92.

8. Dutta, Prajit, (1999): Strategies and Games: Theory and Practice, MIT Price. 
9. Fisher, Ronald, (1930): The Genetical Theory of Natural Selection. Clarendon Press, Oxford.

10. Fudenberg, Drew y Jean Tirole(1991), Game Theory, MIT Press, ISBN 0262061414.

11. Friedman, J.W.(1991): Teoría de Juegos con Aplicaciones a la Economía. Editorial alianza Universidad.

12. Ginits, Herbert (2000): Game Theory Evolving. Princeton Univeristy Press, ISBN 0691009430.

13. Harsanyi, J.C., (1953) Cardinal Utility in Welfare Economics and in the Theory of Risk Taking, Journal of Political Economy, 61.

14. Hicks, J.R., (1939): Value and Capital, Clarendon Press, Oxford.

15. Houthakker, H.S., (1950): Revealed Preference and the Utility Function, Econometrica 17.

16. Kreps, D.M.(1994) : Teoría de los Juegos y Modelación Económica. Fondo de cultura Económica, $1^{\circ}$ edición.

17. Majundar, T., (1956) Choice and Revealed Preference, Econometrica, 24.

18. Mishan, E.J., (1956) An Investigation into Some Alleged Contradictions in Welfare Economics, Economic Journal 67.

19. Morgestern, Oskar y John Von Neuman, (1947): The Theory of Games and Economic Behavior. Princeton Univeristy Press.

20. Nash, John, (1950): Equilibrium Points in n-person Games, Porceedings of the national Academy of the USA, 36(1):48-49.

21. Poundstone William: (2005): El Dilema del Prisionero, Alianza Editorial.Madrid

22. Samuelson, P.A., (1947): Foundation of Economics Analysis, Harvard University Press, Cambridge, Mass.

23. Simon Herbert, (1987): Scientific Discovery: Computional Explorations of the Creative Processes, with Pat Langgley, Gary Bradshaww and Jan Zytkow, MIT Press.

24. Smith Maynard, John, (1982) : Evolution and the Theory of Game. Cambridge University Press.

25. Sen, A. K., (1963): Distribution, Transitivity and Little's Welfare Criteria, Economic Journal, 73.

26. Villacís, J., (1993): La Teoría Combinatoria Aplicada a la Teoría de la Utilidad. Esic Market, n 79. Madrid.

27. (1994): La Teoría Combinatoria Aplicada al Estudio de la Producción, Esic Market, pp 43-58.

28. (2004): Chaos and Combinatorial Ordering in Economics: Chaos and a System's Energy Are Similar Concepts. Conference, $6^{\text {th }}$ August in Istambul.

29. (2005): Business, Combinatorial Theory and Decision Making, The Business Review, Cambridge,volume 3, December, pp 55-60. 